\title{
GREGARIOUS KITE FACTORIZATION OF TENSOR PRODUCT OF COMPLETE GRAPHS
}

\author{
A. TAMil ElakkiYA \\ Department of Mathematics \\ Gobi Arts \& Science College, Erode \\ Tamil Nadu, India \\ e-mail: elakki.1@gmail.com \\ AND \\ A. Muthusamy \\ Department of Mathematics \\ Periyar University, Salem \\ Tamil Nadu, India \\ e-mail: ambdu@yahoo.com
}

\begin{abstract}
A kite factorization of a multipartite graph is said to be gregarious if every kite in the factorization has all its vertices in different partite sets. In this paper, we show that there exists a gregarious kite factorization of $K_{m} \times K_{n}$ if and only if $m n \equiv 0(\bmod 4)$ and $(m-1)(n-1) \equiv 0(\bmod 2)$, where $\times$ denotes the tensor product of graphs.
\end{abstract}

Keywords: tensor product, kite, decomposition, gregarious factor, factorization.

2010 Mathematics Subject Classification: 05C70.

\section{REFERENCES}

[1] I. Anderson, Combinatorial Designs and Tournaments (Oxford University Press Inc., New York, 1997).

[2] R.J.R. Abel, C.J. Colbourn and J.H. Dinitz, Mutually orthogonal latin squares $(M O L S)$, chapter in The Handbook of Combinatorial Designs, Second Edition, C.J. Colbourn and J.H. Dinitz, Eds., Discrete Math. Appl. (Chapman \& Hall/CRC Press, Boca Raton, New York, 2007) 160-163. 
[3] J.C. Bermond and J. Schönheim, $G$-decompositions of $K_{n}$, where $G$ has four vertices or less, Discrete Math. 19 (1977) 113-120. doi:10.1016/0012-365X(77)90027-9

[4] C.J. Colbourn, A.C.H. Ling and G.Quattrocchi, Embedding path designs into kite systems, Discrete Math. 297 (2005) 38-48. doi:10.1016/j.disc.2005.04.014

[5] C.M. Fu, Y.F. Hsu, S.W. Lo and W.C. Huang, Some gregarious kite decompositions of complete equipartite graphs, Discrete Math. 313 (2013) 726-732. doi:10.1016/j.disc.2012.10.017

[6] M. Gionfriddo and S. Milici, On the existence of uniformly resolvable decompositions of $K_{v}$ and $K_{v}-I$ into paths and kites, Discrete Math. 313 (2013) 2830-2834. doi:10.1016/j.disc.2013.08.023

[7] L. Gionfriddo and C.C. Lindner, Nesting kite and 4-cycle systems, Australas. J. Combin. 33 (2005) 247-254.

[8] R. Häggkvist, Decompositions of complete bipartite graphs, in: Surveys in Combinatorics, Lond. Math. Soc. Lecture Note Ser. 141 (1989) 115-147. doi:10.1017/CBO9781107359949.007

[9] S. Kucukcifci and C.C. Lindner, The metamorphosis of $\lambda$-fold block designs with block size four into $\lambda$-fold kite systems, J. Combin. Math. Combin. Comput. 40 (2002) 241-252.

[10] C.C. Lindner and C.A. Rodger, Design Theory (Chapman \& Hall/CRC Press, Taylor \& Francis Group, Boca Raton, 2009).

[11] G. Lo Faro and A. Tripodi, The Doyen-Wilson theorem for kite systems, Discrete Math. 306 (2006) 2695-2701. doi:10.1016/j.disc.2006.03.074

[12] G. Ragusa, Complete simultaneous metamorphosis of $\lambda$-fold kite systems, J. Combin. Math. Combin. Comput. 73 (2010) 159-180.

[13] D.K. Ray-Chaudhuri and R.M. Wilson, Solution of Kirkman's schoolgirl problem, Combinatorics, Proc. Symp. Pure Math., Amer. Math. Soc. 19 (1971) 187-204. doi:10.1090/pspum/019/9959

[14] A. Tamil Elakkiya and A. Muthusamy, $P_{3}$-factorization of triangulated Cartesian product of complete graphs, Discrete Math. Algorithms Appl. 7 (2015) ID:1450066. doi:10.1142/S1793830914500669

[15] A. Tamil Elakkiya and A. Muthusamy, Gregarious kite decomposition of tensor product of complete graphs, Electron. Notes Discrete Math. 53 (2016) 83-96. doi:10.1016/j.endm.2016.05.008

[16] A. Tamil Elakkiya and A. Muthusamy, $P_{3}$-factorization of triangulated Cartesian product of complete graph of odd order, S. Arumugam et al. (Eds.), ICTCSDM 2016, Lecture Notes in Comput. Sci. 10398 (2017) 425-434.

doi:10.1007/978-3-319-64419-6_54 
[17] L. Wang, On the existence of resolvable $\left(K_{3}+e\right)$-group divisible designs, Graphs Combin. 26 (2010) 879-889.

doi:10.1007/s00373-010-0954-5

[18] H. Wang and Y. Chang, Kite-group divisible designs of type $g^{t} u^{1}$, Graphs Combin. 22 (2006) 545-571. doi:10.1007/s00373-006-0681-0

[19] H. Wang and Y. Chang, $\left(K_{3}+e, \lambda\right)$-group divisible designs of type $g^{t} u^{1}$, Ars Combin. 89 (2008) 63-88.

Received 13 March 2017

Revised 14 November 2017

Accepted 19 December 2017 